\title{
Analysis of Tissue Phantom Ratio of the Megavoltage Photon Beams
}

\author{
M. A. Rahman ${ }^{1^{*}}$, M. Jahangir Alam ${ }^{2}$ \\ ${ }^{1}$ Department of Arts \& Sciences, AUST, Dhaka, BANGLADESH \\ ${ }^{2}$ Ahsania Mission Cancer \& General Hospital, Uttara, Dhaka, BANGLADESH \\ *Email for Correspondence: mdashrafur.as@aust.edu
}

\begin{abstract}
Iso-centric beam data, phantom tissue ratios (TPR) are a dosimetric quantity commonly used to describe the change in dose with depth in tissue. Measurement of this is time-consuming and has the possibility of lose the consistency. The value of this quantity of any filed size in any depth is possible to calculate conveniently by the newly developed formula using only percentage depth dose (PDD) data of two fields. PDD for square fields ranging from 2 to $30 \mathrm{~cm}$ and various depths in increment of $0.4 \mathrm{~cm}$ up to maximum $30 \mathrm{~cm}$ have been measured in water at a fixed source surface distance (SSD) of $90 \mathrm{~cm}$ for 4, 6 and 15 MV photon beams in Ahsania Mission Cancer \& General Hospital (AMCGH), Dhaka, Bangladesh. TPR values calculating for these energies of the same field sizes, depths and SSD by using the developed formula compared with those determined from the measured PDD data using a standard formula and had the good agreement. Mean error less than $1 \%$ observed between these TPR values.
\end{abstract}

Key words: TPR, PDD, Dosimetry, Photon beam and Radiation Measurement

\begin{tabular}{ll}
\hline $\mathbf{3 / 8 / 2 0 1 6}$ & Source of Support: None, No Conflict of Interest: Declared \\
\hline $\begin{array}{l}\text { This article is is licensed under a Creative Commons Attribution-NonCommercial 4.0 International License. } \\
\text { Attribution-NonCommercial (CC BY-NC) license lets others remix, tweak, and build upon work non-commercially, and although the new works must also } \\
\text { acknowledge \& be non-commercial. }\end{array}$
\end{tabular}

\section{INTRODUCTION}

There are several radiation dosimetry quantities such as percentage depth dose (PDD), tissue phantom ratio (TPR), tissue maximum ratio (TMR), tissue air ratio (TAR) and backscatter factor (BSF) (Purdy J A 1977, Rahman M. A. et al 2016). TPR is being used to describe the change in dose with depth in tissue. Data for different field sizes of this quantity are often used as reference data check determined within an individual radiotherapy department (Neil Richmond and Robert Brackenridge 2014).These data are usually tabulated as a function of depth and field size at the iso-centre for a given quality index. Most of the tabulated data are calculated from measured PDD of open field central axis even though all these dosimetry quantities can be determined empirically (Bjärngard BE, Zhu TC, Ceberg C 1996). It is often convenient to calculate dose per monitor unit using iso-centric beam data based on TPR values than the measured radiation beam data in the form of PDD.

TPR is formed by the ratio of two doses:

$T P R=D_{d} / D_{r e f}$

where $D_{\text {ref }}$ is the dose at a specific reference point on the central axis at a fixed reference depth, $d_{\text {ref, }}$ in water and $D_{d}$ is the dose measured at an arbitrary depth where TPR value wants to be known. The reference point is often defined at $100 \mathrm{~cm}$ from the source which is typically the source to axis distance (SAD) and $\mathrm{d}_{\mathrm{ref}}$ can be chosen at any depth but $10 \mathrm{~cm}$ is common.

Although TPR data can be measured directly to avoid uncertainties associated with deriving them from percentage depth dose but in practice, they are generally converted from PDD curves because it is convenient to measure PDD at a fixed source to surface distance (Das I J et al. 1996, Sharma S C et al. 2007). This is because many water tank systems do not have the ability to change accurately the depth of water while leaving the chamber at a fixed SSD. 
Furthermore, manually changing the depth of water for many depths and field sizes is very time-consuming and often unpractical (Alam M J et al. 2007, Narayanasamy G et al. 2015].

The accuracy of TPR could affect the monitor unit (MU) calculation (Bjarngard BE, Bar-Deroma R, Corrao A 1994). Knowledge of the dependence of the quantity on various parameters including energy, field size and depth is essential to provide accurate dose and MU (G. X Ding and Rob Krauss 2013). Commissioning of a treatment planning system (TPS) depends on the accuracy of the beam data of TPR (Bedford JL et al. 2003). Data for different field sizes and depths of the quantity of 4, 6 and $15 \mathrm{MV}$ photons have been calculated using the newly developed formula (Alam M J et al. 2007). The advantage of the formula is that the value of the quantity of any field size and any depth can be obtained easily by using measured PDD values of two fields. TPR values obtained from the equation (1) compared with those calculated using the newly developed formula in this work.

\section{MATERIAL AND METHOD}

It was intended to analysis the dosimetry quantity Tissue-Phantom Ratio of high energy photon beams of diverse range for treatment of cancer patient. This quantity usually calculated from the measured percentage depth dose data. The doses were measured in water as it is always assumed to be the better phantom for being very close to human due to its density and number of electrons per gram and universally available with reproducible radiation properties.

Measurements for PDD were made using a PTW water tank using Mephisto $\mathrm{mc}^{2}$ software version 1.3 on an Elekta Linear Accelerator aligned to the central axis of the radiation beam. Detector alignment was achieved by scanning cross plane and in plane profiles for each field size at $10 \mathrm{~cm}$ deep, $90 \mathrm{~cm}$ source-surface distance (SSD). The data recorded from the water surface to $30 \mathrm{~cm}$ deep in 0.4 increments for square collimator setting from $30 \times 30 \mathrm{~cm}^{2}$ to $2 \times$ $2 \mathrm{~cm}^{2}$ for 4,6 , and $15 \mathrm{MV}$ photon beams. TPRs were determined by using these measured PDDs in the equation (1). PDD values of two field sizes $10 \times 10 \mathrm{~cm}^{2}$ and $30 \times 30 \mathrm{~cm}^{2}$ were used in the developed formula (Alam M J et al. 2007) to calculate TPRs for 7 square fields 2, 5, 10.15, 20, 25 and $30 \mathrm{~cm}$ for depths for the same photon beams up to $30 \mathrm{~cm}$ in 0.4 increments. Each TPR data normalized at a depth of $10 \mathrm{~cm}$.

\section{Result And Discussion}

The values of tissue-phantom ratio (TPR) calculated for three photon energies, 4, 6, and $15 \mathrm{MV}$ of 7 field sizes ranging from $2 \mathrm{~cm}$ to $30 \mathrm{~cm}$ and various depths up to $30 \mathrm{~cm}$ with the increment of $0.4 \mathrm{~cm}$ along the central beam axis using a newly developed formula (Alam M J et al. 2007) where PDD values of two field sizes used. A comparison study performed between these calculated values normalized to $10 \mathrm{~cm}$ depth and TPR values determined from measured PDD data using the standard equation (1). The parameters that characterize the TPR curve including the $d_{\max }$ and surface were tabulated for the seven square field sizes in the table 1 to 6 for the energies.

Table 1: TPRs calculated from the developed formula in water at SSD $90 \mathrm{~cm}$ for $4 \mathrm{MV}$ photon beam.

\begin{tabular}{|c|c|c|c|c|c|c|}
\hline Field Size $\left(\mathrm{cm}^{2}\right)$ & $5 \times 5$ & $10 \times 10$ & $15 \times 15$ & $20 \times 20$ & $25 \times 25$ & $30 \times 30$ \\
\hline Depth in (cm) & TPR & TPR & TPR & TPR & TPR & TPR \\
\hline 0 & 0.741 & 0.765 & 0.787 & 0.808 & 0.831 & 0.850 \\
\hline 1.2 & 1.367 & 1.342 & 1.315 & 1.289 & 1.266 & 1.242 \\
\hline 2 & 1.356 & 1.330 & 1.305 & 1.279 & 1.256 & 1.233 \\
\hline 4 & 1.274 & 1.256 & 1.236 & 1.218 & 1.200 & 1.183 \\
\hline 6 & 1.186 & 1.174 & 1.161 & 1.148 & 1.137 & 1.125 \\
\hline 8 & 1.093 & 1.087 & 1.081 & 1.0754 & 1.071 & 1.064 \\
\hline 10 & 1 & 1 & 1 & 1 & 1 & 1 \\
\hline 12 & 0.912 & 0.918 & 0.922 & 0.927 & 0.931 & 0.935 \\
\hline 14 & 0.832 & 0.8425 & 0.850 & 0.858741 & 0.868 & 0.874 \\
\hline 16 & 0.755 & 0.768 & 0.780 & 0.791 & 0.802 & 0.812 \\
\hline 18 & 0.686 & 0.703 & 0.716 & 0.730 & 0.743 & 0.756 \\
\hline 20 & 0.626 & 0.641 & 0.657 & 0.671 & 0.686 & 0.700 \\
\hline 22 & 0.565 & 0.583 & 0.600 & 0.616 & 0.633 & 0.648 \\
\hline 24 & 0.514 & 0.534 & 0.550 & 0.566 & 0.583 & 0.598 \\
\hline 26 & 0.468 & 0.487 & 0.504 & 0.520 & 0.537 & 0.552 \\
\hline 28 & 0.427 & 0.445 & 0.4627 & 0.4783 & 0.494 & 0.509 \\
\hline 30 & 0.3857 & 0.404 & 0.422 & 0.439 & 0.456 & 0.470 \\
\hline
\end{tabular}


Table 2: TPRs determined from measured PDD using equation (1) in water at SSD $90 \mathrm{~cm}$ for 4 MV photon beam

\begin{tabular}{|c|c|c|c|c|c|c|}
\hline Field Size $\left(\mathrm{cm}^{2}\right)$ & $5 \times 5$ & $10 \times 10$ & $15 \times 15$ & $20 \times 20$ & $30 \times 30$ & \\
\hline Depth in $(\mathrm{cm})$ & TPR & TPR & TPR & TPR & TPR & TPR \\
\hline 0 & 0.931 & 0.940 & 0.950 & 0.987 & 1.010 & 1.036 \\
\hline 1.2 & 1.712 & 1.605 & 1.547 & 1.515 & 1.492 & 1.474 \\
\hline 2 & 1.667 & 1.565 & 1.510 & 1.478 & 1.455 & 1.439 \\
\hline 4 & 1.486 & 1.415 & 1.377 & 1.35 & 1.335 & 1.324 \\
\hline 6 & 1.308 & 1.269 & 1.244 & 1.228 & 1.217 & 1.209 \\
\hline 8 & 1.143 & 1.130 & 1.119 & 1.110 & 1.105 & 1.103 \\
\hline 10 & 1 & 1 & 1 & 1 & 1 & 1 \\
\hline 12 & 0.873 & 0.884 & 0.894 & 0.9 & 0.902 & 0.904 \\
\hline 14 & 0.7616 & 0.781 & 0.795 & 0.804 & 0.810 & 0.817 \\
\hline 16 & 0.664 & 0.688 & 0.707 & 0.719 & 0.729 & 0.735 \\
\hline 18 & 0.580 & 0.608 & 0.628 & 0.643 & 0.655 & 0.663 \\
\hline 20 & 0.510 & 0.537 & 0.558 & 0.574 & 0.586 & 0.597 \\
\hline 22 & 0.445 & 0.473 & 0.496 & 0.515 & 0.526 & 0.5383 \\
\hline 24 & 0.392123 & 0.418 & 0.442 & 0.459 & 0.473 & 0.4837 \\
\hline 26 & 0.344 & 0.370 & 0.3931 & 0.410 & 0.423 & 0.435 \\
\hline 28 & 0.303 & 0.329 & 0.349 & 0.366 & 0.379 & 0.390 \\
\hline 30 & 0.267 & 0.290 & 0.311 & 0.327 & 0.340 & 0.352 \\
\hline
\end{tabular}

Table 3: TPRs calculated from the developed formula in water at SSD $90 \mathrm{~cm}$ for $6 \mathrm{MV}$ photon beam

\begin{tabular}{|c|c|c|c|c|c|c|c|}
\hline Field Size $\left(\mathbf{c m}^{2}\right)$ & $2 \times 2$ & $5 \times 5$ & $10 \times 10$ & $15 \times 15$ & $20 \times 20$ & $25 \times 25$ & \multicolumn{1}{l|}{$30 \times 30$} \\
\hline Depth in cm & TPR & TPR & TPR & TPR & TPR & TPR & TPR \\
\hline 0 & 0.688 & 0.679 & 0.709 & 0.760 & 0.802 & 0.844 & 0.875 \\
\hline 1.6 & 1.291 & 1.279 & 1.261 & 1.244 & 1.228 & 1.212 & 1.195 \\
\hline 2 & 1.298 & 1.284 & 1.265 & 1.247 & 1.229 & 1.212 & 1.194 \\
\hline 4 & 1.236 & 1.224 & 1.210 & 1.195 & 1.182 & 1.168188 & 1.152 \\
\hline 6 & 1.159 & 1.151 & 1.142 & 1.132 & 1.124 & 1.114 & 1.105 \\
\hline 8 & 1.079 & 1.074 & 1.070 & 1.066 & 1.063 & 1.059 & 1.055 \\
\hline 10 & 1 & 1 & 1 & 1 & 1 & 1 & 1 \\
\hline 12 & 0.925 & 0.927 & 0.930 & 0.935 & 0.939 & 0.942 & 0.946 \\
\hline 14 & 0.852 & 0.856 & 0.864 & 0.871 & 0.879 & 0.886 & 0.892 \\
\hline 16 & 0.786111 & 0.792 & 0.801 & 0.812 & 0.821 & 0.831 & 0.840 \\
\hline 18 & 0.725 & 0.731 & 0.744 & 0.756 & 0.767 & 0.779 & 0.789 \\
\hline 20 & 0.668 & 0.675 & 0.689 & 0.702 & 0.715 & 0.727 & 0.739 \\
\hline 22 & 0.615 & 0.624 & 0.639 & 0.653 & 0.667 & 0.680 & 0.692 \\
\hline 24 & 0.566 & 0.574 & 0.590 & 0.605 & 0.620 & 0.634 & 0.647 \\
\hline 26 & 0.525 & 0.533 & 0.548 & 0.563 & 0.578 & 0.591 & 0.605 \\
\hline 28 & 0.481 & 0.491 & 0.506 & 0.522 & 0.536 & 0.551 & 0.565 \\
\hline 30 & 0.444 & 0.453 & 0.469 & 0.484 & 0.499 & 0.513 & 0.526 \\
\hline
\end{tabular}

Table 4: TPRs determined from measured PDD using equation (1) in water at SSD $90 \mathrm{~cm}$ for $6 \mathrm{MV}$ photon beam

\begin{tabular}{|c|c|c|c|c|c|c|c|}
\hline Field Size $\left(\mathrm{cm}^{2}\right)$ & $2 \times 2$ & $5 \times 5$ & $15 \times 15$ & $20 \times 20$ & $25 \times 25$ & $30 \times 30$ & \\
\hline Depth in $\mathrm{cm}$ & TPR & TPR & TPR & TPR & TPR & TPR & TPR \\
\hline 0 & 0.637 & 0.665 & 0.711 & 0.756 & 0.799 & 0.8420 & 0.883 \\
\hline 1.6 & 1.538 & 1.525 & 1.503 & 1.483 & 1.463 & 1.443 & 1.424 \\
\hline 2 & 1.533 & 1.519 & 1.496 & 1.474 & 1.452 & 1.431 & 1.410 \\
\hline 4 & 1.397 & 1.386 & 1.369 & 1.353 & 1.337 & 1.321 & 1.306 \\
\hline 6 & 1.256 & 1.250 & 1.239 & 1.229 & 1.219 & 1.209 & 1.199 \\
\hline 8 & 1.122 & 1.119 & 1.115 & 1.111 & 1.106 & 1.102 & 1.098 \\
\hline 10 & 1 & 1 & 1 & 1 & 1 & 1 & 1 \\
\hline 12 & 0.888 & 0.891 & 0.894 & 0.898 & 0.902 & 0.906 & 0.909 \\
\hline 14 & 0.788 & 0.792 & 0.799 & 0.806 & 0.812 & 0.818 & 0.825 \\
\hline 16 & 0.699 & 0.705 & 0.714 & 0.723 & 0.731 & 0.739 & 0.748 \\
\hline 18 & 0.621 & 0.627 & 0.638 & 0.648 & 0.658 & 0.667 & 0.677 \\
\hline 20 & 0.552 & 0.558 & 0.569 & 0.580 & 0.591 & 0.601 & 0.611 \\
\hline 22 & 0.490 & 0.497 & 0.509 & 0.520 & 0.531 & 0.542 & 0.552 \\
\hline 24 & 0.435 & 0.442 & 0.454 & 0.466 & 0.477 & 0.487 & 0.498 \\
\hline 26 & 0.389 & 0.396 & 0.407 & 0.418 & 0.429 & 0.439 & 0.449 \\
\hline 28 & 0.345 & 0.352 & 0.363 & 0.374 & 0.385 & 0.395 & 0.406 \\
\hline 30 & 0.308 & 0.315 & 0.326 & 0.336 & 0.346 & 0.356 & 0.366 \\
\hline
\end{tabular}


Table 5: TPRs calculated from the developed formula in water at $90 \mathrm{~cm}$ SSD for $15 \mathrm{MV}$ photon beam

\begin{tabular}{|c|c|c|c|c|c|c|c|}
\hline Field Sizes $\left(\mathrm{cm}^{2}\right)$ & $2 \times 2$ & $5 \times 5$ & $10 \times 10$ & $20 \times 20$ & $25 \times 25$ & $30 \times 30$ & \\
\hline Depth in $\mathrm{cm}$ & TPR & TPR & TPR & TPR & TPR & TPR & TPR \\
\hline 0 & 0.354 & 0.357 & 0.454 & 0.515 & 0.600 & 0.649 & 0.691 \\
\hline 1.2 & 0.932 & 0.942 & 0.957 & 0.973 & 0.990 & 1.007 & 1.023 \\
\hline 2 & 1.100 & 1.101 & 1.100 & 1.101 & 1.103 & 1.104 & 1.105 \\
\hline 2.8 & 1.390 & 1.349 & 1.329 & 1.317 & 1.313 & 1.314 & 1.307 \\
\hline 4 & 1.159 & 1.154 & 1.145 & 1.137 & 1.129 & 1.122 & 1.114 \\
\hline 6 & 1.110 & 1.106 & 1.099 & 1.094 & 1.089 & 1.084 & 1.079 \\
\hline 8 & 1.056 & 1.055 & 1.051 & 1.047 & 1.045 & 1.042 & 1.040 \\
\hline 10 & 1 & 1 & 1 & 1 & 1 & 1 & 1 \\
\hline 12 & 0.948 & 0.949 & 0.950 & 0.953 & 0.954 & 0.957 & 0.959 \\
\hline 14 & 0.894 & 0.897 & 0.901 & 0.905 & 0.910 & 0.914 & 0.919 \\
\hline 16 & 0.846 & 0.849 & 0.854 & 0.861 & 0.867 & 0.872 & 0.879 \\
\hline 18 & 0.795 & 0.800 & 0.807 & 0.815 & 0.823 & 0.831 & 0.839 \\
\hline 20 & 0.753 & 0.758 & 0.765 & 0.775 & 0.784 & 0.793 & 0.801 \\
\hline 22 & 0.708 & 0.714 & 0.723 & 0.733 & 0.743 & 0.752 & 0.763 \\
\hline 24 & 0.669 & 0.675 & 0.685 & 0.696 & 0.705 & 0.716 & 0.726 \\
\hline 26 & 0.631 & 0.638 & 0.648 & 0.659 & 0.669 & 0.680 & 0.691 \\
\hline 28 & 0.596 & 0.601 & 0.613 & 0.624 & 0.635 & 0.646 & 0.656 \\
\hline 30 & 0.563 & 0.569 & 0.579 & 0.590 & 0.601 & 0.612 & 0.623 \\
\hline
\end{tabular}

Table 6: TPRs determined from measured PDD using equation (1) in water at SSD $9 \mathrm{~cm}$ for $15 \mathrm{MV}$ photon beam

\begin{tabular}{|c|c|c|c|c|c|c|c|}
\hline Field Sizes $\left(\mathrm{cm}^{2}\right)$ & $2 \times 2$ & $5 \times 5$ & $15 \times 15$ & $20 \times 20$ & $25 \times 25$ & $30 \times 30$ & \\
\hline Depth in $\mathrm{cm}$ & TPR & TPR & TPR & TPR & TPR & TPR & TPR \\
\hline 0 & 0.354 & 0.357 & 0.454 & 0.515 & 0.600 & 0.649 & 0.691 \\
\hline 1.2 & 1.183 & 1.113 & 1.151 & 1.172 & 1.211 & 1.224 & 1.228 \\
\hline 2 & 1.368 & 1.303 & 1.300 & 1.300 & 1.308 & 1.308 & 1.303 \\
\hline 2.8 & 1.390 & 1.349 & 1.329 & 1.317 & 1.313 & 1.307 & 1.300 \\
\hline 4 & 1.340 & 1.321 & 1.295 & 1.277 & 1.270 & 1.262 & 1.256 \\
\hline 6 & 1.221 & 1.211 & 1.192 & 1.181 & 1.176 & 1.172 & 1.167 \\
\hline 8 & 1.105 & 1.101 & 1.094 & 1.086 & 1.085 & 1.084 & 1.079 \\
\hline 10 & 1 & 1 & 1 & 1 & 1 & 1 & 1 \\
\hline 12 & 0.905 & 0.908 & 0.914 & 0.918 & 0.920 & 0.923 & 0.924 \\
\hline 14 & 0.820 & 0.824 & 0.835 & 0.841 & 0.848 & 0.851 & 0.853 \\
\hline 16 & 0.744 & 0.748 & 0.763 & 0.772 & 0.779 & 0.784 & 0.788 \\
\hline 18 & 0.675 & 0.680 & 0.695 & 0.707 & 0.717 & 0.722 & 0.726 \\
\hline 20 & 0.614 & 0.618 & 0.636 & 0.649 & 0.659 & 0.666 & 0.671 \\
\hline 22 & 0.559 & 0.562 & 0.581 & 0.595 & 0.606 & 0.613 & 0.619 \\
\hline 24 & 0.509 & 0.512 & 0.531 & 0.546 & 0.558 & 0.566 & 0.571 \\
\hline 26 & 0.4631 & 0.466 & 0.486 & 0.500 & 0.513 & 0.521 & 0.526 \\
\hline 28 & 0.422 & 0.426 & 0.445 & 0.461 & 0.470 & 0.480 & 0.486 \\
\hline 30 & 0.385 & 0.388 & 0.408 & 0.422 & 0.434 & 0.442 & 0.448 \\
\hline
\end{tabular}

Fig.1: Distribution of Tissue-phantom ratios along cental axis for different quality photon beams in water

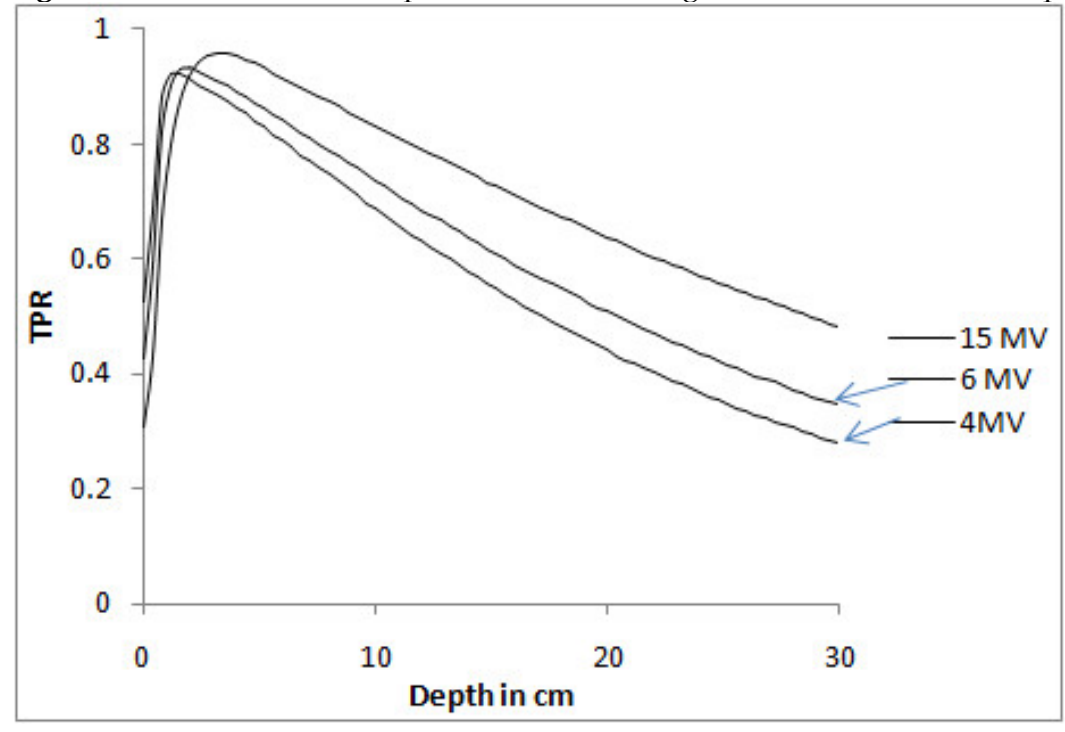


Figure 1 shows that TPR values depend on the depth and energy. It is seen that maximum TPR is not at the surface but at some depths. Beyond the depth of maximum value, it varies exponentially with the depth. The figure also indicates that for all energies it increases rapidly with first few centimeters and then gradually achieves its maximum value at the depth of peak dose. In the case of $4 \mathrm{MV}$ photon beam it occurs at $1.2 \mathrm{~cm}$. Similarly, maximum values occur at $1.6 \mathrm{~cm}$ and $2.8 \mathrm{~cm}$, for 6 and $15 \mathrm{MV}$ photon beams respectively. Higher energy beams have grater penetrating power thus deliver a high depth dose. As a result, the peak value of TPR increases with the energy.

\section{MV Photon Beams}

Fig.2: Comparison of TPRs along the central beam axis in increment of $0.4 \mathrm{~cm}$ up to maximum $30 \mathrm{~cm}$ of various square field sizes of $4 \mathrm{MV}$ photon beam
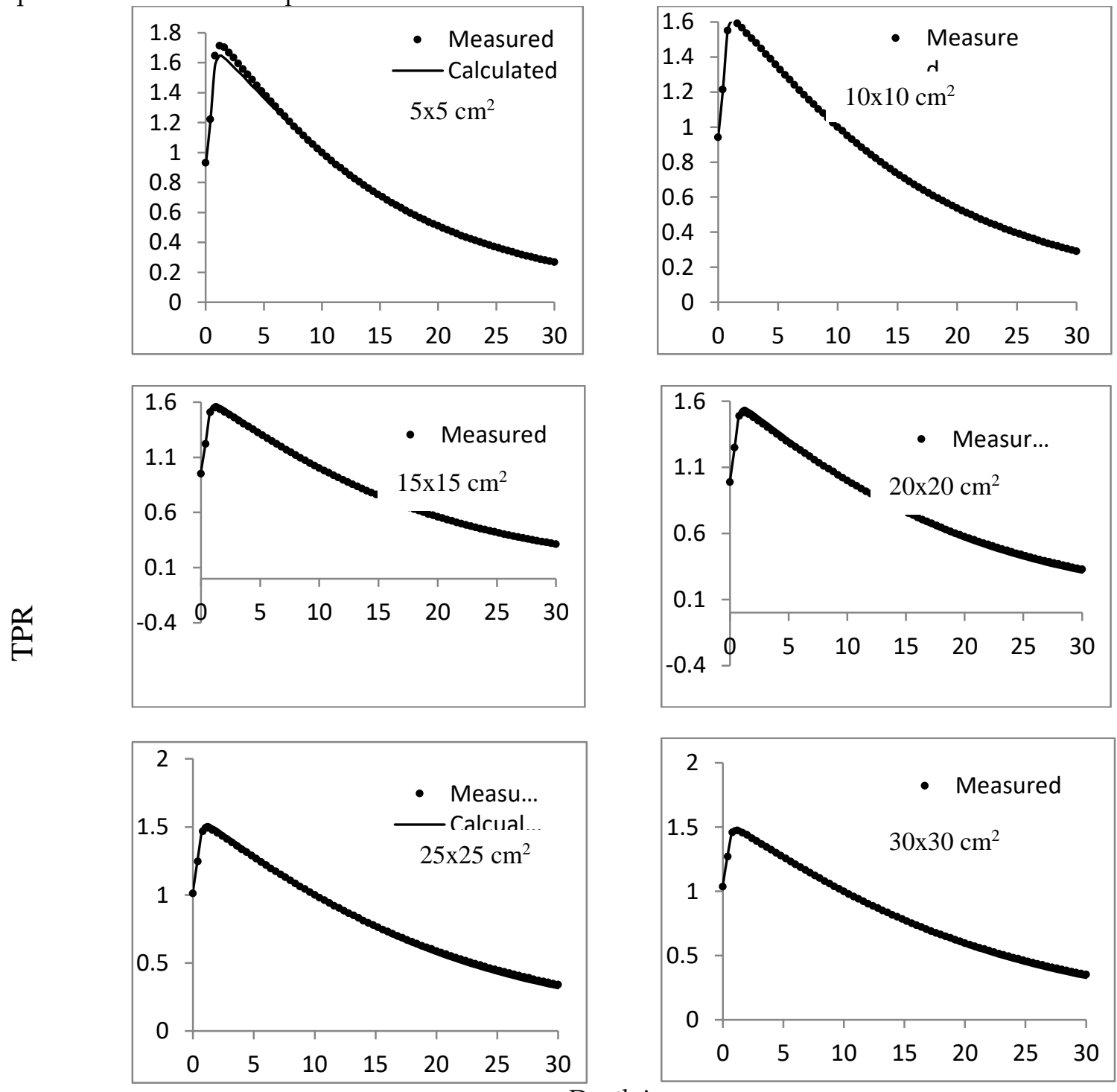

Depth in $\mathrm{cm}$

Figure 2 presents the comparison of TPR values of this energy obtained from both the formulas. The agreement is good for all depths and fields sizes and indicates that TPR values calculated from the developed formula are comparable to those determined from the measured PDD data using the equation (1). Thus, it has the potential to reduce greatly the difficulty and time required to obtain accurate TPR values than directly measuring TPRs. Some TPRs of shown in the figure 2 are listed in Tables 1 and 2 as a function of depth and field size. By comparing these tables, it can be seen that maximum difference occurs by 0.34 at $1.2 \mathrm{~cm}$ depth of the field size $5 \times 5 \mathrm{~cm}^{2}$ and lowest by 0.027 at $12 \mathrm{~cm}$ depth of the field size of $20 \times 20 \mathrm{~cm}^{2}$. When averaged over the entire depth of acquisition, the mean difference in TPR values are $0.6 \%, 0.3 \%, 0.5 \%, 0.6 \%$, and $0.7 \%$ of the field sizes $5,10,15,20,25$ and $30 \mathrm{~cm}^{2}$ respectively. A trend is seen in the Fig.3. 
Fig.3: Percent difference between TPR values with the increasing of field sizes. Decreasing mean difference first with field sizes then increasing but later decreases

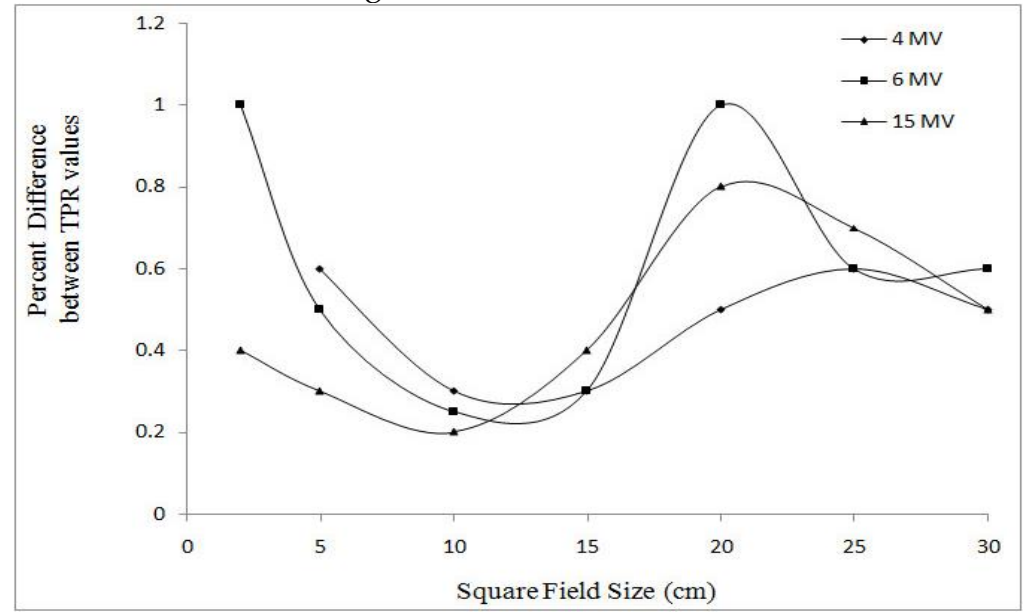

\section{MV Photon BeAms}

Fig.4: Comparison of TPRs along the central beam axis in increment of $0.4 \mathrm{~cm}$ up to maximum $30 \mathrm{~cm}$ of various square field sizes of $6 \mathrm{MV}$ photon beam
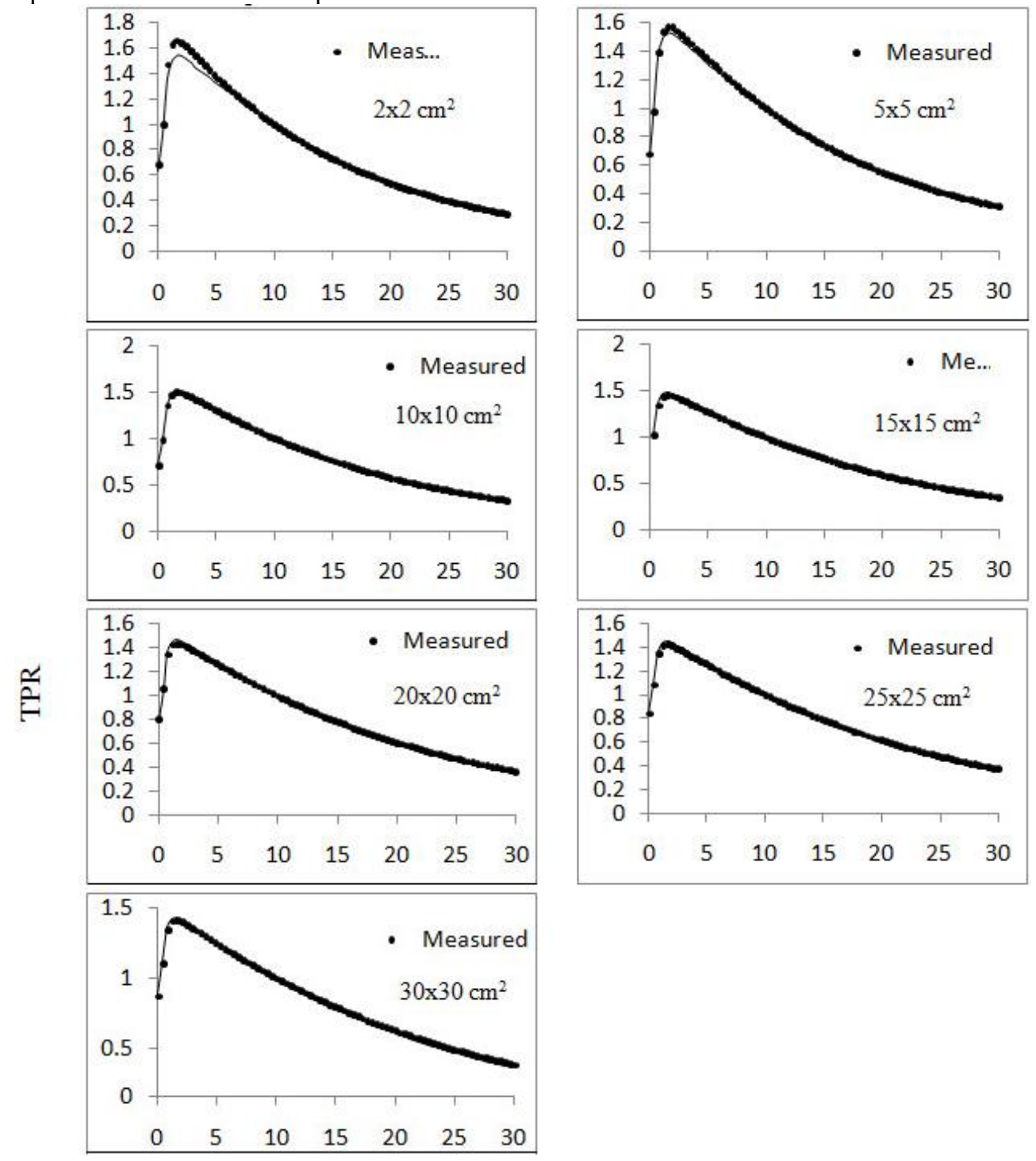

Depth in $\mathrm{cm}$ 
The comparison between TPR values for this energy presented in the Fig.4. The agreement is good for all depths and fields sizes and indicates that TPR values calculated from the developed formula are comparable to those determined from the measured PDD data using the equation (1). Some TPR values of shown in the Fig. 4 are listed in tables 3 and 4 as a function depth and field size. By comparing these Tables, it can be seen that maximum difference occurs by 0.24 at $1.6 \mathrm{~cm}$ depth of field size $2 \times 2 \mathrm{~cm}^{2}$ and lowest by 0.035 at $12 \mathrm{~cm}$ depth of field size of $10 \times 10 \mathrm{~cm}^{2}$. When averaged over the entire depth of acquisition, the mean difference in TPR values are $1 \%, 0.5 \%, 0.2 \%, 0.3 \%$, $1 \%, 0.6 \%$ and $0.5 \%$ of the field sizes $2,5,10,15,20,25$ and $30 \mathrm{~cm}^{2}$ respectively. A trend is seen in the Fig. 3 in decreasing mean difference first with field sizes then increasing but later decreases.

\section{MV Photon Beams}

Fig.5: Comparison of TPRs along the central beam axis in increment of $0.4 \mathrm{~cm}$ up to maximum $30 \mathrm{~cm}$ of various square field sizes of $15 \mathrm{MV}$ photon beam
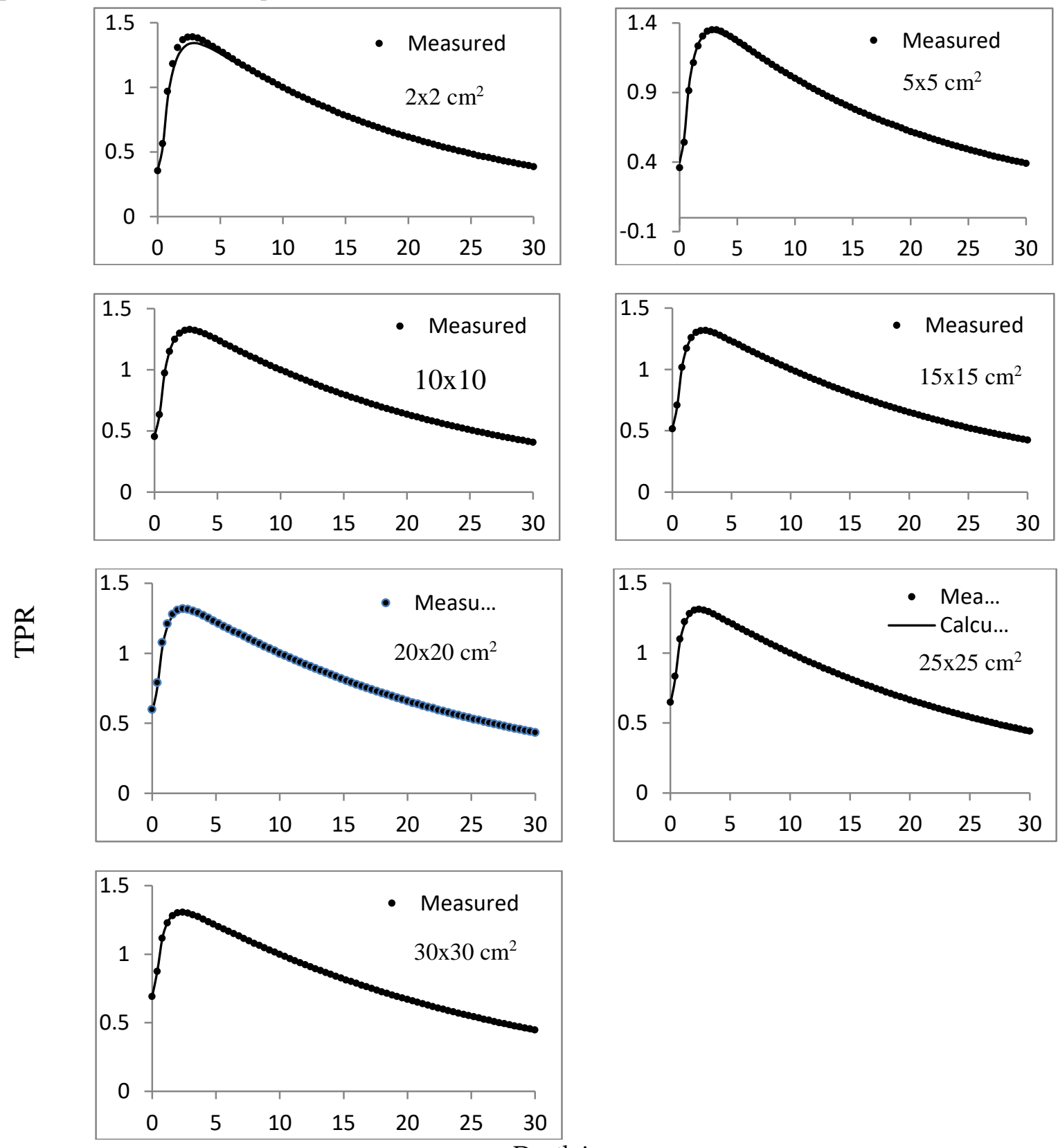

Depth in cm

The comparison of the TPR values presented in the Fig.5 of this energy is in good agreement for all depths and fields sizes and shows both the data are comparable. Some TPR values of shown in the Fig.5 are listed in Tables 5 and 6 as a function depth and field size. By comparing these tables, it can be seen that maximum difference occurs by 0.25 at $1.2 \mathrm{~cm}$ depth of field size $2 \times 2 \mathrm{~cm}^{2}$ and lowest by 0.033 at $12 \mathrm{~cm}$ depth of field size of $20 \times 20 \mathrm{~cm}^{2}$. When averaged over 
the entire depth of acquisition, the mean difference in TPR values are $0.5 \%, 0.3 \%, 0.2 \%, 0.4 \%$, and $0.8 \%, 0.7 \%$ and $0.5 \%$ of the field sizes $2,5,10,15,20,25$ and $30 \mathrm{~cm}^{2}$ respectively. A trend is seen in the Fig. 3 in decreasing mean difference first with field sizes then increasing but later also decreases.

\section{Conclusion}

Calculation of TPR data for seven square field sizes from the developed formula using PDDs of two fields has been shown to have good agreement with those determined from the measured PDDs using a standard formula (1). The mean difference in TPR values averaged over the entire depth 0 to $30 \mathrm{~cm}$ is less than $1 \%$ and shows that these values obtained from the developed formula has the potential to reduce greatly the difficulty and time required to obtain accurate TPRs and would be suitable for clinical use for all clinically relevant depths and field sizes.

\section{ACKNOWLEDGEMENT}

The authors would like to acknowledge the Director, Ahsania Mission Cancer and General Hospital, Uttara, Dhaka, Bangladesh for giving opportunity one of the authors to work there. Thanks to all technical staff of the Hospital for cooperation throughout the process.

\section{REFERENCES}

Bedford JL, Childs PJ, Nordmark Hansen V et al. Commissioning and quality assurance of the Pinnacle(3) radiotherapy treatment planning system for external beam photons. Br J Radiol 2003; 76: 163-76

Bjarngard BE, Bar-Aroma R, Corrao A. A survey of methods to calculate monitor settings. Int J Radiat Oncol Biol Phys 1994; $28: 749$

Bjärngard BE, Zhu TC, Cyborg C.Tissue-phantom ratio and percentage depth doses. Med phys1996; $23: 629$.

Das I J et al. Characteristics of a dedicated linear accelerator-based stereotactic radiosurgery -radiotherapy unit Radiotherapy, Oncol1996; 38: 61-8.

G. X Ding and Rob Krauss. An empirical formula to obtain tissue-phantom ratios from and percentage depth dose curves for small fields. Phys. Med. Biol 2013; 58: 4781.

Ganesh Narayanasamy, Wilbert Cruz, Niko Papanikolaou, Sotirios Stathakis. TPR comparison with \& without PSF correction. Int. J. Cancer ther Oncol 2015; 3; 3024.

M. Jahangir Alam et al. A modified formula for defining tissue phantom ratio of photon beams. Bangladesh Med. Coun Bull 2007; 33: 92-97.

Neil Richmond and Robert Brackenridge. A comparison of small-field tissue phantom ratio data generation methods for an Elekta Agility 6 MV photon beam. Medical Dosimetry 2014; 39: 60-63.

Purdy J A. Relationship between tissue-phantom ratio and percentage depth dose. Med phys1977; 4: 66-7.

Rahman M A, M. Jahangir Alam and M. Akhtaruzzaman, Characteristics analysis of high energy external radiotherapy beams in water. Malays. j. med. biol. Res 2016. Accepted, 3:1

Sharma S C et al. J. Appl. Clin. Med. Phys 2007; 8: 119-125 